\title{
The self regulating star formation of gas rich dwarf galaxies in quiescent phase
}

\author{
M. A. R. Kobayashi and H. Kamaya
}

\author{
Department of Astronomy, School of Science, Kyoto University, Sakyo-ku, Kyoto 606-8502, Japan \\ e-mail: kobayasi@kusastro.kyoto-u.ac.jp
}

Received 2 July 2004 / Accepted 26 August 2004

\begin{abstract}
The expected episodic or intermittent star formation histories (SFHs) of gas rich dwarf irregular galaxies (dIrrs) are the longstanding puzzles to understand their whole evolutional history. Solving this puzzle, we should grasp what physical mechanism causes the quiescent phase of star formation under the very gas rich condition after the first starburst phase. We consider that this quiescent phase is kept by lack of $\mathrm{H}_{2}$, which can be important coolant to generate the next generation of stars in the low-metal environment like dIrrs. Furthermore, in dIrrs, $\mathrm{H}_{2}$ formation through gas-phase reactions may dominate the one on dust-grain surfaces because their interstellar medium (ISM) are very plentiful and the typical dust-to-gas ratio of dIrrs ( $D_{\text {dIrrs }}=1.31 \times 10^{-2} D_{\mathrm{MW}}$, where $D_{\mathrm{Mw}}$ is its value for the local ISM) is on the same order with a critical value $D_{\text {cr }} \sim 10^{-2} D_{\mathrm{Mw}}$. We show that the lack of $\mathrm{H}_{2}$ is mainly led by $\mathrm{H}^{-}$destruction when gas-phase $\mathrm{H}_{2}$ formation dominates since $\mathrm{H}^{-}$is important intermediary of gas-phase $\mathrm{H}_{2}$ formation. $\mathrm{H}^{-}$is destroyed by the radiation from all stars born in the previous starburst phase because $\mathrm{H}^{-}$destroying infrared photon can penetrate the whole ISM of dIrrs. Considering the physical process which timescale is the longest as main process in regulating global star formation, we can show this lack of $\mathrm{H}_{2}$ leads the quiescent phase of star formation. Hence, we can say that the stellar radiation which destroys $\mathrm{H}^{-}$and leads low $\mathrm{H}_{2}$ abundance should be properly treated in studying SFHs of dIrrs.
\end{abstract}

Key words. galaxies: dwarf - galaxies: evolution - galaxies: irregular - stars: formation

\section{Introduction}

Dwarf galaxies are the galaxies with lower luminosity, lower mass and smaller size, and they are much more abundant in number than normal galaxies on the Hubble sequence like our Milky Way (see Kunth \& Östlin 2000, for a recent review of local group dwarf galaxies). These natures imply that they are basic building blocks of the normal galaxies in hierarchical structure formation scenarios of the cold dark matter universe (Cole et al. 2000; Corbin \& Vacca 2002; Monica 2003). In addition, the dwarf galaxies have lower abundances of heavy elements than those of the normal galaxies; therefore, they are still in an early stage of chemical evolution and/or are analogies to primordial galaxies (e.g., Izotov \& Thuan 1999).

Dwarf galaxies are classified by their morphological appearances into some basic types. One of the morphological types is dwarf irregular galaxies (dIrrs), which characteristics are irregular and amorphous appearances at optical wavelengths. Interestingly, unlike other morphological types of dwarf galaxies, dIrrs still have plentiful interstellar medium (ISM) and in general show ongoing star formation and HII region (Mateo 1998; Kunth \& Östlin 2000). The star formation histories (SFHs) of dIrrs are considered to be episodic, that is, quiescent phase follows a starburst phase (Greggio et al. 1993; Tolstoy et al. 1998; Grebel 1999), and their star formation rates (SFRs) are more widely distributed $\left(\sim 10^{-4}-10^{-2}\left[M_{\odot} / \mathrm{yr} / \mathrm{kpc}^{2}\right]\right)$ than that of the normal galaxies (Hunter 1997). In this issue, we particularly focus on the dIrrs which are considered to be in their quiescent phase of star formation (i.e. SFR $\sim 10^{-4}\left[M_{\odot} / \mathrm{yr} / \mathrm{kpc}^{2}\right]$ ).

However, despite their closeness, the regulation of star formation even in the Local Group dIrrs is not yet well understood. Actually, it is very surprising that they have been forming stars recently because their gravitational potential are shallow (Skillman \& Bender 1997, for a review) and comparable to the energies of some supernova explosions; therefore, they should have little ISM after some supernovae (several million years from the epoch of dIrrs formed), and thus, the recent and ongoing star formation would not occur. Why do they still have plentiful gas? Why do they show ongoing star formation? Both the questions are still controversial. Even if these questions are solved, another puzzle remains; why do they form stars at such low rates in spite of having plentiful ISM as material of stars? This paper attempts to solve this puzzle by investigating the non-chemical equilibrium molecule formation history of dIrrs on some assumptions.

For the star formation processes in low-metallicity environment, hydrogen molecules $\left(\mathrm{H}_{2}\right)$ can be important coolants at the gas temperature $T \lesssim 10^{4} \mathrm{~K}$ (Peebles \& Dicke 1968); more abundant $\mathrm{H}_{2}$ leads gas to cool faster, and therefore, would 
result in more active star formation. Although $\mathrm{H}_{2}$ forms primarily on the surfaces of dust grains in local molecular clouds, $\mathrm{H}_{2}$ can also form in gas-phase through the reactions, which intermediaries are negatively charged hydrogen $\left(\mathrm{H}^{-}\right)$,

$\mathrm{H}+\mathrm{e}^{-} \rightarrow \mathrm{H}^{-}+\gamma$

$\mathrm{H}^{-}+\mathrm{H} \rightarrow \mathrm{H}_{2}+\mathrm{e}^{-}$,

or ionized hydrogen molecule $\left(\mathrm{H}_{2}^{+}\right)$,

$\mathrm{H}+\mathrm{H}^{+} \rightarrow \mathrm{H}_{2}^{+}+\gamma$

$\mathrm{H}_{2}^{+}+\mathrm{H} \rightarrow \mathrm{H}_{2}+\mathrm{H}^{+}$.

These gas-phase reactions are active $T \sim 10^{4} \mathrm{~K}$ and the reactions (1) and (2) can more efficiently form $\mathrm{H}_{2}$ than the (3) and (4) reactions (e.g., Abel et al. 1997). On the other hand, $\mathrm{H}_{2}$ is dissociated by collisions with other chemical species and the absorption of ultraviolet (UV) photons called the LymanWerner band (LW) photons $(11.26 \mathrm{eV} \leq h v \leq 13.6 \mathrm{eV})$, which are mainly radiated by early type stars (Field et al. 1966).

Many astrophysicists have built the theoretical models concentrated on the dissociation of $\mathrm{H}_{2}$ by the LW photons because they have investigated the second epoch of star formation after the first stars have been formed in metal-free molecular cloud (e.g., Silk 1977; Nishi \& Tashiro 2000). These models result in the low abundance of $\mathrm{H}_{2}$ in relatively large region (several hundred parsec around the source star of the LW photons), and thus, negative-feedback on further star formation in the host cloud of the star. However, in dIrrs, the region affected by LW photons would be smaller than previously predicted because dIrrs are gas rich system; the mean free paths of LW photons are on the order of $1 \mathrm{pc}$ («the typical size of dIrrs) with typical parameters of primordial clouds (Haiman et al. 1996). Furthermore, early type stars as the sources of LW photons are rare in dIrrs. Therefore, the negative-feedback on further star formation brought about the $\mathrm{H}_{2}$ dissociation by the LW photons may be negligible in dIrrs.

By the way, if $\mathrm{H}_{2}$ is formed by gas-phase reactions (Eqs. (1)-(4)), the formative abundance of $\mathrm{H}_{2}, y_{\mathrm{H}_{2}}$, largely depends on the $\mathrm{H}^{-}$abundance, $y_{\mathrm{H}^{-}}$, because the $\mathrm{H}_{2}$ formation process through $\mathrm{H}^{-}$is more effective than the one through $\mathrm{H}_{2}^{+}$ (in Sect. 2, we discuss that gas-phase $\mathrm{H}_{2}$ formation may dominate grain-catalyzed $\mathrm{H}_{2}$ formation in dIrrs). We expect that $y_{\mathrm{H}_{2}}$ in dIrrs is more effectively influenced by the destruction of $\mathrm{H}^{-}$than by the direct dissociation of $\mathrm{H}_{2}$ due to the LW photons. This is because that the typical mean free path of the $\mathrm{H}^{-}$destroying infrared (IR) photons (its wavelength $\lambda \lesssim 1.64 \mu \mathrm{m}), l_{\mathrm{IR}}$, would be sufficiently longer than that of the LW photons; $l_{\mathrm{IR}} \sim 100 \mathrm{kpc}$ ( $\gg$ the typical size of dIrrs) with typical parameters of primordial clouds. Therefore, the IR photons can penetrate and affect the whole ISM. Furthermore, the IR photons are also radiated by low-mass stars as well as early type stars. Thus, all stars born in the previous starburst phase can be considered as the sources of the $\mathrm{H}^{-}$destroying photons. If $\mathrm{H}^{-}$is seriously destroyed by the IR photons of the interstellar radiation field (ISRF) of dIrrs, the star formation activity may become more inactive at almost the whole ISM.

With this expectation, we construct the theoretical model to investigate $y_{\mathrm{H}_{2}}$. In Sect. 2, we compare the rates of gas-phase and grain-catalyzed $\mathrm{H}_{2}$ formation, and show gas-phase $\mathrm{H}_{2}$ formation may dominate in dIrrs. Section 3 describes our model for the star formation process in dIrrs and Sect. 4 presents our results. Finally, we summarize our study and briefly discuss about the next starburst phase in Sect. 5 .

\section{Gas-phase and grain-catalyzed $\mathrm{H}_{2}$ formation}

$\mathrm{H}_{2}$ formation on dust-grain surfaces completely dominates gas-phase one in the local ISM. However, if the dust-to-gas ratio, $D$, is lower than a critical value, $D_{\text {cr }}$, gas-phase $\mathrm{H}_{2}$ formation dominates grain-catalyzed formation (Kamaya \& Hirashita 2001; Glover 2003). Despite the $\mathrm{H}_{2}$ formation rate on grain surfaces is still uncertain, Glover (2003) calculates $D_{\text {cr }}$ in some astrophysical situations by tentatively adopting the rate of Hollenbach \& McKee (1979). As noted in Glover (2003), the temperature dependence of $D_{\text {cr }}$ is very strong; at low temperatures $\left(T \lesssim\right.$ a few $\left.\times 10^{2} \mathrm{~K}\right)$, grain-catalyzed $\mathrm{H}_{2}$ formation is relatively efficient and $D_{\mathrm{cr}}$ is very small $\left(D_{\mathrm{cr}} \lesssim 10^{-3} D_{\mathrm{MW}}\right.$, where $D_{\mathrm{MW}}$ is the dust-to-gas ratio in the local ISM), while at high temperatures, gas-phase $\mathrm{H}_{2}$ formation is efficient and thus, $D_{\mathrm{cr}}$ is large. In addition, $D_{\mathrm{cr}}$ also depends on the ionisation degree and the density of ISM; both high-ionisation and high-density lead the high efficiency of gas-phase $\mathrm{H}_{2}$ formation and large $D_{\mathrm{cr}}$.

By the way, we focus on the process that the ISM of dIrrs once is heated up by stellar-radiation, supernovae and/or other processes, and then cools down to the temperature at which star formation is effective under ISRF. Thus, re-calculating $D_{\text {cr }}$ (Eq. (35) of Glover 2003) by using the physical parameters in which we interested, we have $D_{\text {cr }} \gtrsim 10^{-2} D_{\text {MW }}$ at $T \gtrsim 5000 \mathrm{~K}$ as shown in the dotted line of Fig. 1. This critical value is on the same order of magnitude with the typical value for dIrrs $D_{\mathrm{dIrrs}} \sim 1.3 \times 10^{-2} D_{\mathrm{MW}}$ (Lisenfeld \& Ferrara 1998).

At $T \lesssim 5000 \mathrm{~K}$, the ISM cools off at a stroke. This is because $D_{\text {cr }}$ becomes smaller than $D_{\text {dirrs }}$, and thus, $\mathrm{H}_{2}$ formation on dust-grain surfaces may dominate; therefore, $y_{\mathrm{H}_{2}}$ rapidly increases. Furthermore, if the metallicity of dIrrs is higher than a critical value $\sim 10^{-2} Z_{\odot}$ (Nishi \& Tashiro 2000) (the same order with the metallicity of the most metal-deficient galaxy, I $\mathrm{Zw}$ 18), metal cooling dominates $\mathrm{H}_{2}$ cooling at this temperature range. However, we are interested in the temperature at which the cooling timescale is the longest because global star formation timescale is decided by the slowest physical process. Thus, the interesting temperature is found to be $\sim 5000 \mathrm{~K}$, at which gas-phase $\mathrm{H}_{2}$ formation dominates. Therefore, it is meaningful to study the SFHs of dIrrs on the assumption that gas-phase $\mathrm{H}_{2}$ formation is important.

\section{Model and calculation}

We calculate the time-dependent, non-chemical equilibrium, ionisation and molecule formation history of a gas system in dIrrs composed of hydrogen and helium. Neglecting the dynamics of the gas system in ISM, we concentrate on the time evolution of the density and temperature in some regions in dIrrs. We consider the following 9 chemical species; 


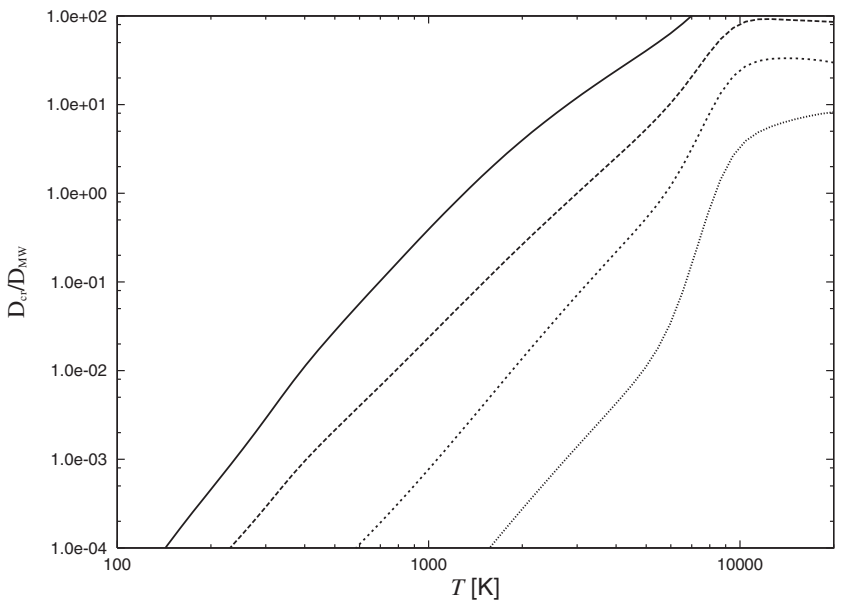

Fig. 1. $D_{\mathrm{cr}}$ as a function of the gas temperature $T$ under some ISRF intensities are presented. The thick, long-dashed, short-dashed and dotted lines represent $D_{\text {cr }}$ for the ISRF intensity ratios $\varepsilon$ of $0.010,0.10$, 1.0 , and 10.0, respectively. The ionisation degree and the density are self-consistently calculated.

$\mathrm{H}, \mathrm{H}^{+}, \mathrm{H}^{-}, \mathrm{H}_{2}, \mathrm{H}_{2}^{+}, \mathrm{He}, \mathrm{He}^{+}, \mathrm{He}^{++}, \mathrm{e}^{-}$. In dIrrs, the helium abundance is still maintained almost primordial value because dIrrs are still in an early stage of chemical evolution; therefore, it is assumed to be primordial value ( $24 \%$ of hydrogen by mass fraction) in our calculation (Skillman et al. 1993; Izotov et al. 1999).

At first, the ISRF in our model, $J_{v}$, is assumed to have same energy dependence with that of the solar vicinity, $J_{v}^{\odot}$ (see Mathis et al. 1983), and its intensity is normalised by that of the solar vicinity; $J_{v}=\varepsilon \cdot J_{v}^{\odot}$. In order to investigate the dependence of $y_{\mathrm{H}_{2}}$ on the ISRF, we change the ratio $\varepsilon$ from 0.01 to 100 with the step of one order of magnitude. At next, decomposing the ISRF of solar vicinity into four parts, that is, early type stars, two types of disc stars $\left(T_{\text {eff }}=7500 \mathrm{~K}, 4000 \mathrm{~K}\right)$ and red giant stars $\left(T_{\text {eff }}=3000 \mathrm{~K}\right)$ as in Mathis et al. (1983), we investigate which degree each component affects $y_{\mathrm{H}_{2}}$. Initial conditions of all calculations are $T_{\text {ini }}=1.0 \times 10^{6} \mathrm{~K}, n_{\text {ini }}=3.0 \times 10^{-3} \mathrm{~cm}^{-3}$, and the initial abundances of each chemical species are that in chemical equilibrium at $T_{\text {ini }}$.

\section{Results}

Figure 2 shows $y_{\mathrm{H}_{2}}$ and $y_{\mathrm{H}^{-}}$under some ISRF intensities. As the ISRF becomes more intense, these formative abundances monotonically decrease at a fixed temperature. $y_{\mathrm{H}_{2}}$ at $T=$ $5000 \mathrm{~K}$, which is tightly connected with the SFRs of dIrrs in our model (see Eq. (6)), reaches $\sim 10^{-5}$ for the ISRF ten times more intense than that of the solar vicinity. This low $\mathrm{H}_{2}$ abundance means that the ISM cannot cool down and that star formation is delayed, and thus, this can explain the observed small SFRs of dIrrs in their quiescent phase.

The dependences of $y_{\mathrm{H}_{2}}$ at $5000 \mathrm{~K}$ on the ISRF intensity are drawn in Fig. 3. The radiation from both disc stars $\left(T_{\mathrm{eff}}=\right.$ $7500 \mathrm{~K}, 4000 \mathrm{~K})$ and giant stars $\left(T_{\text {eff }}=3000 \mathrm{~K}\right)$ is as important as that from early type stars as the LW band photon sources in order to decrease $y_{\mathrm{H}_{2}}$. The ISRF intensity, which is slightly stronger than that of solar vicinity, may be easily attainable

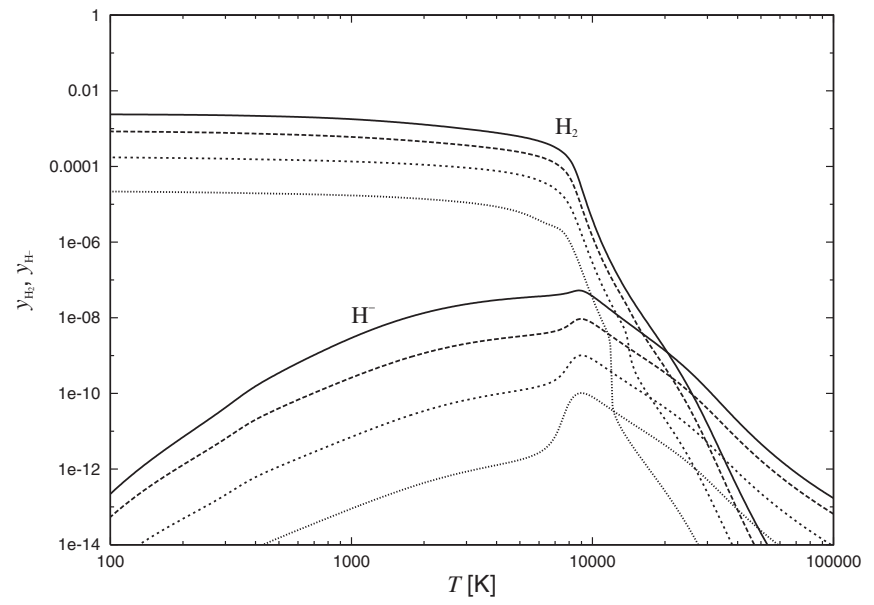

Fig. 2. $y_{\mathrm{H}_{2}}$ and $y_{\mathrm{H}^{-}}$as a function of $T$ under some ISRF intensities are presented. The four lines increased monotonically as the temperature decreases are $y_{\mathrm{H}_{2}}$ (labelled as " $\mathrm{H}_{2}$ ") and other four lines are $y_{\mathrm{H}^{-}}$(also labelled as " $\mathrm{H}^{-}$"). The thick, long-dashed, short-dashed and dotted lines represent the formative abundances for the ISRF intensity ratios $\varepsilon$ of $0.010,0.10,1.0$, and 10.0 , respectively.

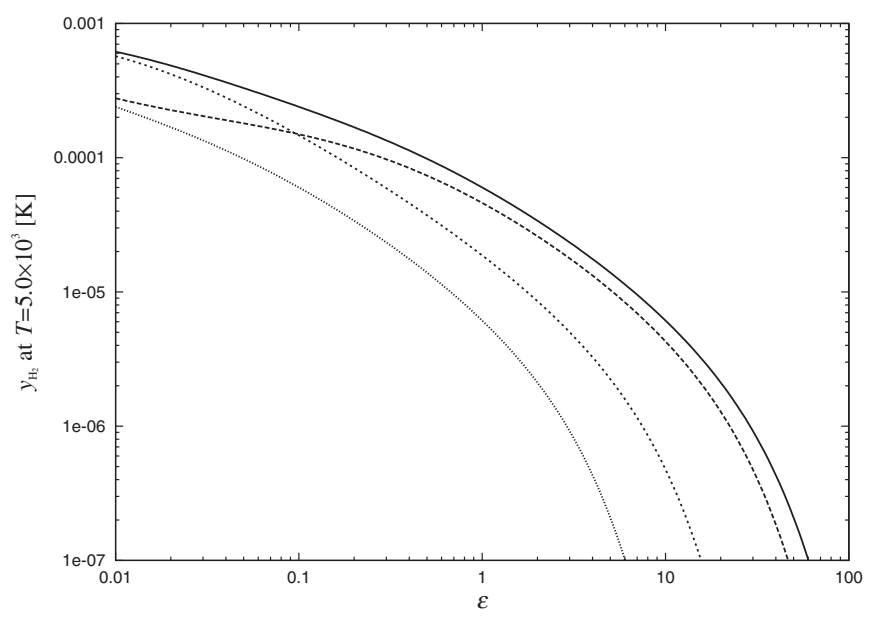

Fig. 3. $y_{\mathrm{H}_{2}}$ at $T=5000 \mathrm{~K}$ as a function of the normalized ISRF intensity, $\varepsilon$, and its dependence on the spectral energy distribution (SED) of ISRF are shown. The thick line represents $y_{\mathrm{H}_{2}}$ for the SED of the solar vicinity ISRF. The long-dashed line is also the one, while the ISRF has increment of all components except for early type stars by an order of magnitude. The short-dashed is that for the ISRF with increment of only the component of early type stars by an order of magnitude. The dotted line is for the all components to be increased by an order of magnitude.

because the ISRF intensity of the solar vicinity is still much weaker than that of the Magellanic Clouds (Bluhm \& de Boer 2001; Contursi et al. 2000).

Here, we simply model the SFR, using Jeans mass, $M_{\mathrm{J}}$, Jeans radius, $r_{\mathrm{J}}$, cooling time, $t_{\mathrm{cool}}$, and the mass conversion fraction from clouds to stars (star formation efficiency; SFE);

$\mathrm{SFR}=\frac{\mathrm{SFE} \cdot M_{\mathrm{J}}}{t_{\mathrm{cool}}} / r_{\mathrm{J}}^{2}$

This equation means that a cloud with mass $M_{\mathrm{J}}$ and size $r_{\mathrm{J}}$ will cool down in the timescale $t_{\text {cool }}$ and some portions of the cloud 


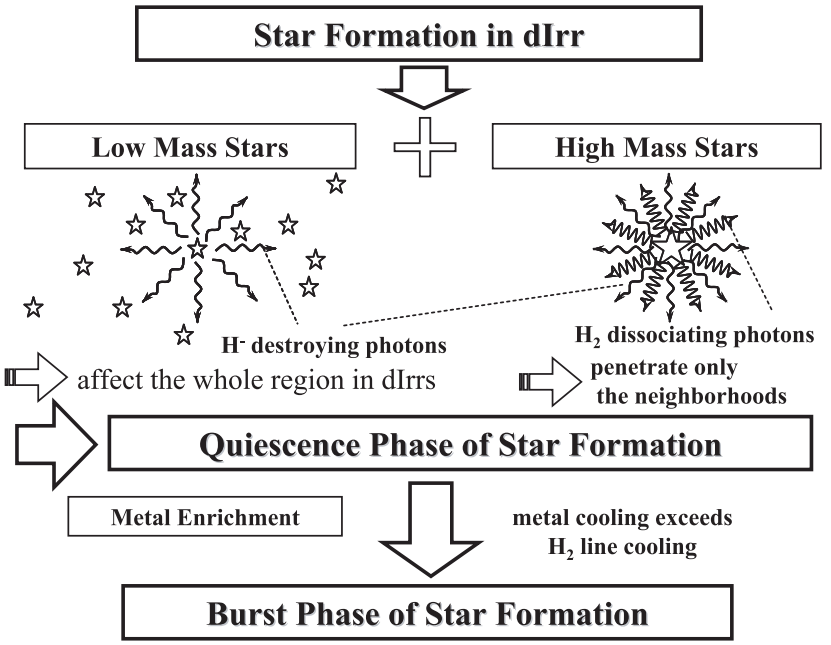

Fig. 4. The schematic explanation of our scenario for SFHs of dIrrs.

which mass fraction are represented by SFE will convert into stars. Then, we apply the model to our simulation, and get

$\mathrm{SFR} \sim 1.0 \times 10^{-4} \cdot\left(\frac{\mathrm{SFE}}{10^{-3}}\right) \cdot\left(\frac{y_{\mathrm{H}_{2}}}{10^{-5}}\right)\left[M_{\odot} / \mathrm{yr} / \mathrm{kpc}^{2}\right]$,

where $y_{\mathrm{H}_{2}}$ is the abundance of $\mathrm{H}_{2}$ at $5000 \mathrm{~K}$, at which the cooling timescale is the longest. We can predict that the SFE of dIrrs is low (the order of $10^{-3}$ ), and ISRF of dIrrs is stronger than that of the solar vicinity in order to understand why the SFRs of dIrrs are such low.

\section{Summary and discussion}

We can conclude that the low mass stars, which are considered to be $\mathrm{H}^{-}$destroying photon sources, are as important as high mass OB stars as the sources of LW band photon in understanding the episodic SFHs of dIrrs. That is, the radiation from many low mass stars burn in previous burst epoch of star formation destroys $\mathrm{H}^{-}$, which is the intermediary of gas-phase $\mathrm{H}_{2}$ formation process, and suppresses further $\mathrm{H}_{2}$ formation with $\mathrm{H}_{2}$ dissociation, and keep the SFHs in quiescent phase. The schematic explanation of our conclusion is represented by Fig. 4. Incidentally, we can say that low mass stars are more important than $\mathrm{OB}$ stars because the typical life time of OB stars is considerably short $\left(\sim 10^{6} \mathrm{yr}\right)$. Furthermore, low mass stars may be much more abundant in low surface brightness galaxies than ordinary expected (Lee et al. 2004); that is, "bottom-heavy" initial mass function (IMF) is suggested there. If this IMF stands up in dIrrs, the contribution of the low mass stars to decrease $y_{\mathrm{H}_{2}}$ may become more and more important.

Acknowledgements. We are grateful to the referee for his/her encouraging comments. This work is supported by the Grant-in-Aid from the Ministry of Education, Culture, Sports, Science and Technology (MEXT) of Japan (16740110) and the Grant-in-Aid for the 21st Century COE "Center for Diversity and Universality in Physics" from MEXT of Japan.

\section{References}

Abel, T., Anninos, P., Zhang, Y., \& Norman, M. L. 1997, New Astron., 2,181

Bluhm, H., \& de Boer, K. S. 2001, A\&A, 379, 82

Cole, S., Lacey, C., Baugh, C. M., \& Frenk, C. S. 2000, MNRAS, 319, 168

Contursi, A., Lequeux, J., Cesarsky, D., et al. 2000, A\&A, 362, 310

Corbin, M. R., \& Vacca, W. D. 2002, ApJ, 581, 1039

Field, G. B., Somerville, W. B., \& Dressler, K. 1966, ARA\&A, 4, 207

Glover, S. C. O. 2003, ApJ, 584, 331

Grebel, E. K. 1999, in The Stellar Content of the Local Group, ed. P. Whitelock, \& R. Cannon (San Francisco: ASP), IAU Symp., 192, 17

Greggio, L., Marconi, G., Tosi, M., \& Focardi, P. 1993, AJ, 105, 894

Haiman, Z., Rees M. J., \& Loeb, A. 1996, ApJ, 467, 522

Hollenbach, D., \& McKee, C. F. 1979, ApJS, 41, 555

Hunter, D. A. 1997, PASP, 109, 937

Izotov, Y. I., \& Thuan, T. X. 1999, ApJ, 511, 639

Izotov, Y. I., Chaffee, F. H., Foltz, C. B., et al. 1999, ApJ, 527, 757

Kamaya, H., \& Hirashita, H. 2001, PASJ, 53, 483

Kunth, D., \& Östlin, G. 2000, A\&ARv, 10, 1

Lee, H.-c., Gibson, B. K., Flynn, C., Kawata, D., \& Beasley, M. A. 2004, MNRAS, 353, 113

Lisenfeld, U., \& Ferrara, A. 1998, ApJ, 496, 145

Mateo, M. 1998, ARA\&A, 36, 435

Mathis, J. S., Mezger, P. G., \& Panagia, N. 1983, A\&A, 128, 212

Monica, T. 2003, Ap\&SS, 284, 651

Nishi, R., \& Tashiro, M. 2000, ApJ, 537, 50

Peebles, P. J. E., \& Dicke, R. H. 1968, ApJ, 154, 891

Silk, J. 1977, ApJ, 211, 638

Skillman, E. D., \& Bender, R. 1995, Rev. Mex. Astron. Astrofis., 3,

Skillman, E. D., Terlevich, R. J., Terlevich, E., Kennicutt, R. C., Jr., \& Garnett, D. R. 1993, in Texas/PASCOS '92 Relativistic Astrophysics and Particle Cosmology, ed. C. Akerlof, \& M. Srednicki (New York: New York Academy of Sciences), 739 Tolstoy, E., Gallagher. J. S., Cole, A. A., et al. 1998, AJ, 116, 1244 